\title{
Fokus auf frühe Prävention und innovative Intervention
}

\author{
Alexandra Philipsen \\ Klinik und Poliklinik für Psychiatrie und Psychotherapie, Universitätsklinikum Bonn AöR, Bonn, Deutschland
}

Es ist mir eine Freude, Ihnen die letzte Ausgabe der Verhaltenstherapie in diesem Jahr 2019 vorstellen $\mathrm{zu}$ können. Mit dieser Ausgabe spannen wir einen Bogen von Erhebungsinstrumenten und Konzepten zur Verbesserung von Verhaltensauffälligkeiten im Schulkindesalter über den aktuellen Stand zur Anwendung Online-basierter und automatisierter Interventionen (Chatboots) in der Psychotherapie.

Als Mitglied der Steuergruppe bei der Erstellung der S3-Leitlinien ADHS [Arbeitsgemeinschaft der Wissenschaftlichen Medizinischen Fachgesellschaften] erlaube ich mir insbesondere, zu den beiden ersten Arbeiten von Lauth-Lebens und Lauth [2019] "Erfassung von symptomkritischen und belastenden Schulsituationen bei Kindern mit Aufmerksamkeitsdefizit-/Hyperaktivitätsstörung: Schülerauffälligkeiten und Lehrerbelastungen" sowie von Pfeiffer et al. [2019] "Konzeption, Akzeptanz und Durchführbarkeit einer modularisierten universellen Präventionsmaßnahme zur emotionalen Kompetenz für Kinder und Jugendliche an Schulen im Rahmen eines Service-Learning-Konzepts" ausführlicher Stellung zu nehmen.

Ziel der erstgenannten Arbeit war die Erstellung eines deutschsprachigen Inventars für ADHS-bezogene Schülerauffälligkeiten und die damit assoziierte Belastung des Lehrpersonals, welches entsprechend der Empfehlungen der Leitlinien auch als Messinstrument für zukünftige Interventionsstudien dienen kann. Testtheoretisch erweist sich das Instrument als valide und differenziert Schüler und Schülerinnen mit ADHS und solche ohne diagnosti- zierte ADHS, aber mit Verhaltensauffälligkeiten zuverlässig von nicht betroffenen Schülern und Schülerinnen und zeigt einen deutlichen Zusammenhang mit der Belastung der Lehrer. Die Studie führt uns auch nochmals vor Augen, dass insbesondere interaktionelle Situationen nicht nur mit Lehrpersonal, sondern auch mit gleichaltrigen Kindern durch ADHS konflikthaft beladen sein können und damit, ebenso wie das Lernverhalten, Inhalt von entsprechenden Interventionen sein sollten. Entsprechend werden in den Leitlinien Interventionen für Lehrer bei ADHS empfohlen. Als in der Erwachsenenmedizin tätige Psychiaterin und Psychotherapeutin, die mit den häufig negativen psychosozialen Auswirkungen [Lam et al., 2017] einer persistierenden ADHS in das Erwachsenenalter befasst ist, bin ich über weitere hilfreiche Konzepte, die gerade auch den Fokus auf die zwischenmenschlichen Beziehungen bei ADHS legen und möglicherweise mittel- und langfristig präventiv wirksam sein können, dankbar.

Die hohe Akzeptanz und gute Anwendbarkeit einer solchen - modularisierten - Präventionsmaßnahme für Schulen, hier zur transdiagnostischen emotionalen Kompetenz, werden im zweiten Artikel aufgeführt. Dies hat umso höhere Bedeutung, als dass wir wissen, dass die Vulnerabilität für psychische Erkrankungen früh angelegt ist [Anttila et al., 2018], grundlagenbasierte Studien eine starke Überlappung von psychischen Erkrankungen belegen und gerade bei ADHS eine emotionale Instabilität im Kindesalter als ein bedeutsamer Prädiktor für die Persistenz in das Erwachsenenalter belegt ist [Shaw et al.,

\section{KARGER}

(C) 2019 S. Karger AG, Basel
Prof. Dr. med. Alexandra Philipsen

Klinik und Poliklinik für Psychiatrie und Psychotherapie

Universitätsklinikum Bonn AöR, Venusberg-Campus 1

DE-53127 Bonn (Deutschland)

E-Mail sekretariat.psychiatrie@ukbonn.de 
2014]. Der Beweis, dass ein solches Präventionsprogramm jenseits einer guten Durchführbarkeit auch klinisch relevante Ergebnisse im longitudinalen Verlauf erbringt, muss allerdings noch erbracht werden.

Postpartale Depressionen sind häufig und haben erhebliche Auswirkungen auf die Mutter-(Vater-)Kind-Beziehung. Neben der medikamentösen Behandlung ist die kurzfristige Wirksamkeit von psychotherapeutischen in der Regel kognitiv-behavioralen - Therapieprogrammen belegt [Huang et al., 2020]. Trotz hoher Relevanz und nicht ausreichender Verfügbarkeit therapeutischer Unterstützung ist bislang wenig über Online-Programme und deren Nutzen bei dieser Indikation bekannt. Auf den ersten Blick mag dies auch kontraintuitiv erscheinen, da im Fokus der Behandlung die unmittelbare Beziehung zwischen in der Regel der Mutter und dem Kind liegt. Entsprechend wurden die Kolleginnen Westerhoff et al. [2019] auch wenig fündig. Dennoch könnten - analog zu anderen Störungsbildern - entsprechende leicht verfüg- bare und niederschwellige Angebote eine vielversprechende (ergänzende) Therapiemöglichkeit für Mütter mit postpartaler Depression und deren Kindern während, nach oder vielleicht sogar anstelle einer Face-toFace-Intervention darstellen und sollten entsprechend weiter auf Akzeptanz und Wirksamkeit untersucht werden.

Hier schließt sich der Kreis: Depressive mütterliche Symptome sind assoziiert mit Störungen der exekutiven Funktionen im jungen Kindesalter [Park et al., 2018], und diese wiederum sind häufig mit $\mathrm{ADHS}$ vergesellschaftet. Daher gilt es Präventivmaßnahmen noch früher als in der Schule anzusetzen. Das wiederum erfordert eine enge $\mathrm{Zu}$ sammenarbeit von Psychologie und Medizin und deren Fachbereichen.

In diesem Sinne wünsche ich den Leserinnen und Lesern nun eine spannende Lektüre der aktuellen Ausgabe und wünsche allen Kolleginnen und Kollegen einen guten Jahresausklang.

\section{Literatur}

Anttila V, Bulik-Sullivan B, Finucane HK, Walters RK, Bras J, Duncan L, et al.; Brainstorm Consortium. Analysis of shared heritability in common disorders of the brain. Science. 2018 Jun;360(6395):eaap8757.

Arbeitsgemeinschaft der Wissenschaftlichen Medizinischen Fachgesellschaften. S3-Leitlinie: Aufmerksamkeitsdefizit-/Hyperaktivitätsstörung (ADHS) im Kindes-, Jugend- und Erwachsenenalter. https://www.awmf.org/ leitlinien/detail/ll/028-045.html.

Huang R, Yang D, Lei B, Yan C, Tian Y, Huang $\mathrm{X}$, et al. The short- and long-term effectiveness of mother-infant psychotherapy on postpartum depression: a systematic review and meta-analysis. J Affect Disord. 2020 Jan;260: 670-9.
Lam AP, Müller H, Philipsen A. ADHS im Erwachsenenalter - Diagnostik und Therapie. Fortschr Neurol Psychiatr. 2017 Nov;85(11): 696-707.

Lauth-Lebens M, Lauth G. Erfassung von symptomkritischen und belastenden Schulsituationen bei Kindern mit Aufmerksamkeitsdefizit-/Hyperaktivitätsstörung: Schülerauffälligkeiten und Lehrerbelastungen. Verhaltenstherapie. 2019 doi: 10.1159/000498974 [Epub ahead of print].

Park M, Brain U, Grunau RE, Diamond A, Oberlander TF. Maternal depression trajectories from pregnancy to 3 years postpartum are associated with children's behavior and executive functions at 3 and 6 years. Arch Women Ment Health. 2018 Jun;21(3):353-63.
Pfeiffer S, Gutzweiler R, Tschan T, In-Albon T. Konzeption, Akzeptanz und Durchführbarkeit einer modularisierten universellen Präventionsmaßnahme zur emotionalen Kompetenz für Kinder und Jugendliche an Schulen im Rahmen eines Service-LearningKonzepts. Verhaltenstherapie. 2019 doi: 10.1159/000497250 [Epub ahead of print].

Shaw P, Stringaris A, Nigg J, Leibenluft E. Emotion dysregulation in attention deficit hyperactivity disorder. Am J Psychiatry. 2014 Mar; 171(3):276-93.

Westerhoff B, Trösken A, Renneberg B. about:blank? Online Interventions for Postpartum Depression. Verhaltenstherapie. 2019 doi: 10.1159/000501779 [Epub ahead of print]. 